\title{
The approach to scaling in phase-ordering kinetics
}

\author{
C. Castellano ${ }^{1}$ and M. Zannetti ${ }^{2}$ \\ 1 Dipartimento di Scienze Fisiche, Università di Napoli, Mostra d'Oltremare, Padiglione 19, 80125 Napoli, Italy \\ 2 Istituto Nazionale di Fisica della Materia, Unità di Salerno and Dipartimento di Fisica, Università di Salerno, 84081 \\ Baronissi (Salerno), Italy
}

\begin{abstract}
The influence of the initial fluctuations on the onset of scaling in the quench to zero temperature of a two dimensional system with conserved order parameter, is analyzed in detail with and without topological defects. We find that the initial fluctuations greatly affect the way scaling is reached, while the number of components of the order parameter does not play a significant role. Under strong initial fluctuations the preasymptotic linear behavior is replaced by the mean field behavior of the large- $N$ model, producing the multiscaling to standard-scaling crossover also in the physically interesting cases of systems with a small number of components.
\end{abstract}

PACS: 64.60.Cn, 05.70.Fh, 64.60.My, 64.75.+g

Much of the interest in phase ordering-kinetics is devoted to scaling behavior of the structure factor [1]. This is a late stage phenomenon which takes place after the order parameter has reached local equilibrium and the typical domain size $L(t)$ dominates all other lengths. The structure factor obeys the scaling form

$$
C(\vec{k}, t)=L^{d}(t) F(x)
$$

where $x=k L(t), L(t) \sim t^{\frac{1}{z}}$ and, when the order parameter is conserved, $z=3$ or $z=4$ in the scalar or vectorial case, respectively. All what happens before, in the time span between the instant of the quench and the beginning of the late stage, is generically referred to as early stage. Usually the early stage theory is identified with the Cahn-Hilliard linear theory [2] and early stage studies are mainly focused on how and when this theory breaks down [3 6]. From investigations along this line emerges a picture of the early stage as a time regime where quite complex and interesting processes take place. In particular, it appears that the breakdown of the linear theory starts first at short distances and propagates with time towards larger length scales 7 .

Recently [8], the early evolution of the phase-ordering process with a conserved order parameter has been investigated in a different context with the aim of investigating the multiscaling to standard-scaling crossover. In the exact solution of the large- $N$ model [9] the late stage form (1) is replaced by the more general multiscaling behavior

$$
C(\vec{k}, t)=\left[k_{m}^{2-d}(t) L^{2}(t)\right]^{\varphi(x)} F(x)
$$

where $k_{m}(t)$ is the peak wave vector, $x=k / k_{m}, \varphi(x)=$ $\psi(x)$ with $\psi(x) \equiv 1-\left(x^{2}-1\right)^{2}$ and $k_{m} L(t) \sim(\ln t)^{1 / 4}$. It was shown by Bray and Humayun 10] (BH) in the framework of the $1 / N$ expansion that (2) holds as a late stage property only when $N$, the number of the order parameter components, is strictly infinite while the standardscaling form (11) is eventually restored for any finite value of $N$. The full time evolution of the structure factor for $N$ large but finite is then expected to display the crossover from multiscaling to standard-scaling, with a crossover time which grows with $N$ and also with the size $\Delta$ of the fluctuations in the initial state. Indeed, the existence of the crossover together with this dependence of the crossover time on $N$ and $\Delta$ has been found in the numerical solution [8] of the $\mathrm{BH}$ model.

This result suggests to investigate the early behavior of systems with a small number of components and large initial fluctuations, for which no early stage theory is available. In this Letter we will focus on how the size of the fluctuations in the initial condition affects the approach to scaling. The influence of the initial fluctuations on the structure of the early stage was previously pointed out by Elder, Rogers and Desai [4]. Here we are able to make a detailed analysis of this effect and to complete the picture of what happens before scaling is fully developed.

We consider the zero temperature quenches of a twodimensional system described by the Cahn-Hilliard equation

$$
\frac{\partial \vec{\phi}}{\partial t}=-\nabla^{2}\left[\nabla^{2} \vec{\phi}-V^{\prime}(\phi)\right]
$$

with $V(\vec{\phi})=\frac{r}{2} \vec{\phi}^{2}+\frac{g}{4}\left(\vec{\phi}^{2}\right)^{2}$ and $r<0, g>0$ in the two cases of $N=1$ (singular topological defects) and $N=4$ (absence of topological defects). In the initial state the system is uncorrelated

$$
<\vec{\phi}(\vec{x}) \cdot \vec{\phi}\left(\vec{x}^{\prime}\right)>=\Delta \delta\left(\vec{x}-\vec{x}^{\prime}\right)
$$

and $\Delta$ measures the strength of the fluctuations. We will consider the two cases $\Delta \ll \vec{\phi}_{e q}^{2}$ (small $\Delta$ ) and $\Delta \simeq \vec{\phi}_{e q}^{2}$ (large $\Delta$ ), where $\left|\vec{\phi}_{e q}\right|=\sqrt{\frac{-r}{g}}$ is the equilibrium value of the order parameter. We shall find that when $\Delta$ is large the early stage of the phase-ordering process is accurately described by the large- $N$ model (mean field theory), both for $N=1$ and for $N=4$. Then, the multiscaling to standard-scaling crossover is expected to be a generic feature of the early stage, when the order parameter is conserved and fluctuations in the initial state are large. Furthermore, we shall see that the structure of the crossover follows a pattern similar to what one has 
in the transition from linear to nonlinear regime when the initial fluctuations are small [7]. Namely, it turns out that also the mean field theory breakdown takes place through the propagation of the failure from the short to the large length scales.

The tool which allows to monitor all possible behaviors is the measurement of the spectrum of exponents $\varphi(x)$ in (2) over a sequence of time intervals [8]. The form (2) contains (11) as a particular case with $\varphi(x) \equiv 1$. Considering $C(\vec{k}, t)$ in the time interval $\left(t_{0}, \tau\right)$ beginning at $t_{0}$ and of duration $\tau$, after taking the logarithm of Eq.(2) in two dimensions we have

$$
\ln C(\vec{k}, t)=\varphi(\vec{x}) \ln L^{2}(t)+\ln F(x)
$$

which yields $\varphi(x)$ as the slope of $\ln C(\vec{k}, t)$ vs $\ln L^{2}(t)$, over the interval $\left(t_{0}, \tau\right)$, at fixed $x$. In principle, $\varphi(x)$ may depend on $t_{0}$. If this happens scaling does not hold. Instead, if $\varphi(x)$ does not depend on the time interval there is scaling and more specifically there is multiscaling if $\varphi(x)$ depends on $x$, while scaling is standard if $\varphi(x)$ is independent of $x$.

The time evolution of the structure factor has been obtained numerically by simulating the equation of motion with a computationally efficient cell dynamical system 111. The details of the computation are the same of Ref. 12. We used values of the parameters such that that $\vec{\phi}_{e q}^{2}=0.9558$ and the initial amplitudes of the fluctuations were $\Delta=2.083$ (large $\Delta$ ) or $\Delta=8.3 \times 10^{-8}$ $($ small $\Delta)$. The results were averaged over 30 realizations of the initial conditions on a $256 \times 256$ lattice for $N=4$ (small and large $\Delta$ ) and $N=1($ small $\Delta)$. For $N=1$ and large $\Delta$ we used a $512 \times 512$ lattice and averaged over 90 realizations (up to $t=5432$ ) and over 50 realizations (up to $t=54177$ ).

Let us first go over the case of small $\Delta$. In the behavior of $C(\vec{k}, t)$ for $N=1$ (Fig. $\mathbb{1}$ ) one can recognize two sharply separated time regimes, one with $k_{m}$ constant and the other with $k_{m}$ decreasing with time (see also Fig. 6). The latter corresponds to the late stage which displays coarsening and a well formed $k^{-3}$ Porod tail for $k>k_{m}$, indicating the existence of sharp interfaces. For $k<k_{m}$ the structure factor displays the $k^{4}$ power law 14. Interesting non trivial behavior can be observed also in the early stage where $k_{m}$ is constant. Here two subregimes can be identified. At the very beginning, as we shall see more precisely below, the evolution is linear except for the high wave vector region where an incipient Porod tail appears. Later on, but with $k_{m}$ still time independent, the evolution is nonlinear for all $k$ 's due to the appearence of the power law behavior also for $k<k_{m}$. The evolution takes place exactly in the same way for $N=4$, except that the Porod tail is replaced by exponential decay, due to the absence of topological defects 8, 13.

Next, in order to probe the scaling behavior, we have measured $\varphi(x)$ in a sequence of seven time intervals (Fig. 2). The first four curves display a strong dependence of $\varphi(x)$ both on $x$ and on the time interval, while the remaining three, for $t \geq 500$, are essentially flat and time independent. Accordingly, the latter ones belong to the late stage with standard-scaling already established, while the set of the first four clearly illustrates the absence of scaling in the early stage. Furthermore, a closer analysis of the $x$ dependence of these curves provides evidence, also in the case with conserved order parameter, for the propagation of the breaking of linear evolution from high towards low wave vectors, as previously observed in the nonconserved case [7]. In fact, these curves display a minimum which moves towards smaller values of $x$ as time proceeds and the behavior of $\varphi(x)$ to the left of the minimum can be accounted for on the basis of the linearized theory, but not on the right. When $\Delta$ is small at the very beginning the non linear term can be neglected in Eq.(3) and the analytical form of the structure factor can be written as

$$
C(\vec{k}, t)=\Delta \exp \left[2 k_{m}^{4} t \psi(x)\right]
$$

with $k_{m}^{2}=-r / 2$ and $x=k / k_{m}$. Then, taking the logarithm

$$
\ln C(\vec{k}, t)=\left[\frac{2 k_{m}^{4} t}{\ln L^{2}(t)} \psi(x)\right] \ln L^{2}(t)+\ln \Delta
$$

and comparing with Eq.(5) we can identify $\varphi(x)=$ $\frac{2 k_{m}^{4} t}{\ln L^{2}(t)} \psi(x)$. Indeed, the behavior of $\varphi(x)$ to the left of the minimum for curves up to $t \sim 200$ is well fitted by $\psi(x)$ with a growing time dependent prefactor. The behavior of $\varphi(x)$ for $N=4$ is identical except for a slight change in the vertical scale.

The picture changes completely when $\Delta$ is large. The time evolution of $C(\vec{k}, t)$ is displayed in Fig. 3 and the main qualitative differences with Fig. 1 are that there is no regime with constant $k_{m}$ (see also Fig. 6) and that it takes much longer to build up the $k^{4}$ tail for $k<k_{m}$. But the real insight into what is going on is obtained from the behavior of $\varphi(x)$. In Fig. 4 and 5 we have plotted the time evolution of $\varphi(x)$ over six different time intervals. Again $\varphi(x)$ displays a minimum which moves towards lower values of $x$ as time goes on. However, the behavior is much different from what we had with small $\Delta$. Considering the set of the first four curves for $N=1$ (Fig. (4), while on the right of the minimum there is no scaling as before, now on the left there is multiscaling with $\varphi(x)$ time independent and well described by $\psi(x)$ (notice that these curves are magnified by factors of 30 with respect to those in Fig. 2). For larger times (curves from $t \sim 1700$ onward) the opposite occurs, namely on the right of the minimum $\varphi(x)$ tends to lose the time dependence as well as the $x$ dependence, while on the left there appears a time dependence. This overall behavior is quite informative. The set of the first four curves 
shows that in the early stage mean field theory provides an adequate treatment of the nonlinearity all the way down to a certain length scale (corresponding to the minimum in $\varphi(x)$ ), which grows with time. The description of what happens below this cutoff is beyond the reach of mean field theory. Later on $(t \geq 1700)$ mean field theory breaks down over all length scales, since the appearance of a flat behavior together with the disappearance of the time dependence for $x \geq 1$ signals the onset of standard-scaling on the shorter distances, while the time dependence for $x \leq 1$ shows that the crossover towards standard-scaling is under way over larger distances. The $N=4$ (Fig. 同) system behaves essentially in the same way, except that the breakdown of multiscaling for $x \leq 1$ does not show up in the time of the simulation, indicating that the crossover time is larger for $N=4$ than for $N=1$. It is worth stressing that from the above analysis follows that the time regime preceding the fully developed standard-scaling under strong initial fluctuations can be made to last much longer than the corresponding early stage when the initial fluctuations are small.

Additional insight into the difference between small $\Delta$ and large $\Delta$ can be obtained from the behavior of the peak wave vector (Fig. 6). The curves for small $\Delta$ show clearly the existence of the early stage with $k_{m}^{-1}$ constant followed by a sharp transition to power law growth in the scaling regime. Instead, for large $\Delta$ the plateau is replaced by a sharp drop of $k_{m}^{-1}$, soon followed by increasing behavior. This initial decreasing behavior of $k_{m}^{-1}$ and the subsequent change to positive slope are consistent with the solution of the large- $N$ model [9]. Furthermore, the asymptotic slope is reached very slowly. We have analyzed the growth of $k_{m}^{-1}(t)$ with the power law $t^{1 / z}$ and the results for three time intervals are displayed in Table 1. The interesting feature here is that the asymptotic values $1 / 3$ for $N=1$ and $1 / 4$ for $N=4$ are approached from above for small $\Delta$ and from below for large $\Delta$. Thus, in particular, for $N=1$ and $\Delta$ large the effective growth exponent before reaching values close to $1 / 3$ goes through a time region where it is closer to $1 / 4$. This is consistent with the mean field description of the early stage when $\Delta$ is large and shows that the behavior of the effective exponent is quite sensitive to the initial condition for a long time.

In conclusion, we have analyzed the approach to scaling in the quench to zero temperature of a two dimensional system, which may $(N=1)$ or may not $(N=4)$ form stable topological defects, under the effect of weak and strong initial fluctuations. We have found that, for physically interesting systems, the value of $N$ plays no significant role, while the strength of the initial fluctuations is quite important. When $\Delta$ is small there is a clearly identifiable and relatively short early stage, with no scaling, dominated by the formation of domains on a fixed length scale. After these have been formed, coarsening begins, the late stage is entered and dynamics obeys standard-scaling. When $\Delta$ is large, instead, the initial process of domain formation is bypassed and coarsening begins right away, but it is not accompanied by standardscaling. Rather, before standard-scaling sets in there is a long time transient characterized by multiscaling which shows that the early stage evolution is governed by mean field theory. So we are now in the position to make an assessment of what is the proper place for the large- $N$ model in the theory of phase-ordering processes for systems with a small number of components. It is clear, on the basis of the $\mathrm{BH}$ result and of more recent work of Rojas and Bray [13], that the large- $N$ model does not give reliable information on the late stage behavior of these systems, neither the $1 / N$ expansion offers a viable systematic approximation scheme. Instead, as we have found, the large- $N$ model qualifies for the early stage theory, whenever the initial fluctuations are large.

We thank Yoshi Oono for providing the original CDS program from which we have developed ours. One of us (C.C.) whishes also to thank the National Institute of Standards and Technology for the kind hospitality during the completion of this work.

[1] For a recent review see A.J.Bray, Adv. Phys. 43, 357, (1994)

[2] J.W.Cahn and J.E.Hilliard, J. Chem. Phys 31, 688, (1959)

[3] K.Binder, Phys. Rev. A 29, 341, (1984)

[4] K.R.Elder, T.M.Rogers and R.C.Desai, Phys. Rev. A 38, 4725, (1988)

[5] M.Laradji, M.Grant, M.J.Zuckerman and W.Klein, Phys. Rev. B 41, 4646, (1990)

[6] F.Corberi, A.Coniglio and M.Zannetti, Phys. Rev. E 51, 5469, (1995)

[7] N.Gross, W.Klein and K.Ludwig, Phys. Rev. Lett. 73, 2639, (1994); C.Yeung, N.Gross and M.Costolo, Phys. Rev. E 52, 6025, (1995)

[8] C.Castellano and M.Zannetti, Phys. Rev. E 53, 1430, (1996)

[9] A.Coniglio and M.Zannetti, Europhys. Lett. 10, 575, (1989)

[10] A.J.Bray and K.Humayun, Phys. Rev. Lett. 68, 1559, (1992)

[11] Y.Oono and S.Puri, Phys. Rev. Lett. 58, 836, (1987); Phys. Rev. A 38, 434, (1988); S.Puri and Y.Oono, Phys. Rev. A 38, 1542, (1988)

[12] M.Mondello and N.Goldenfeld Phys. Rev. E 47, 2384, (1993)

[13] F.Rojas and A.J.Bray, preprint

[14] C.Yeung, Phys. Rev. Lett. 61, 1135, (1988)

\section{FIGURE CAPTIONS}

1. Time evolution of $C(\vec{k}, t)$ for small $\Delta$ and $N=1$. 
2. Time evolution of $\varphi(x)$ for small $\Delta$ and $N=1$.

3. Time evolution of $C(\vec{k}, t)$ for large $\Delta$ and $N=1$.

4. Time evolution of $\varphi(x)$ for large $\Delta$ and $N=1$.

5. Time evolution of $\varphi(x)$ for large $\Delta$ and $N=4$.

\begin{tabular}{||ll|l|l|l||}
\hline \multicolumn{1}{|c|}{ Time intervals } & \multicolumn{1}{|c|}{$274-1086$} & $1367-5432$ & $6837-54177$ \\
\hline$N=1$ & small $\Delta$ & $0.47 \pm 0.03$ & $0.36 \pm 0.01$ & $0.351 \pm 0.007$ \\
$N=1$ & large $\Delta$ & $0.223 \pm 0.008$ & $0.275 \pm 0.003$ & $0.322 \pm 0.004$ \\
$N=4$ & small $\Delta$ & $0.42 \pm 0.03$ & $0.295 \pm 0.005$ & $0.266 \pm 0.005$ \\
$N=4$ & large $\Delta$ & $0.202 \pm 0.008$ & $0.220 \pm 0.07$ & $0.235 \pm 0.002$ \\
\hline
\end{tabular}

6. Time evolution of the peak wave vector.

\section{TABLE}

Table 1 . Values of the exponent $1 / z$ computed by linear regression on the data displayed in Fig. 6 . 


$$
N=1 \quad \Delta=8.3 \times 10^{-8}
$$

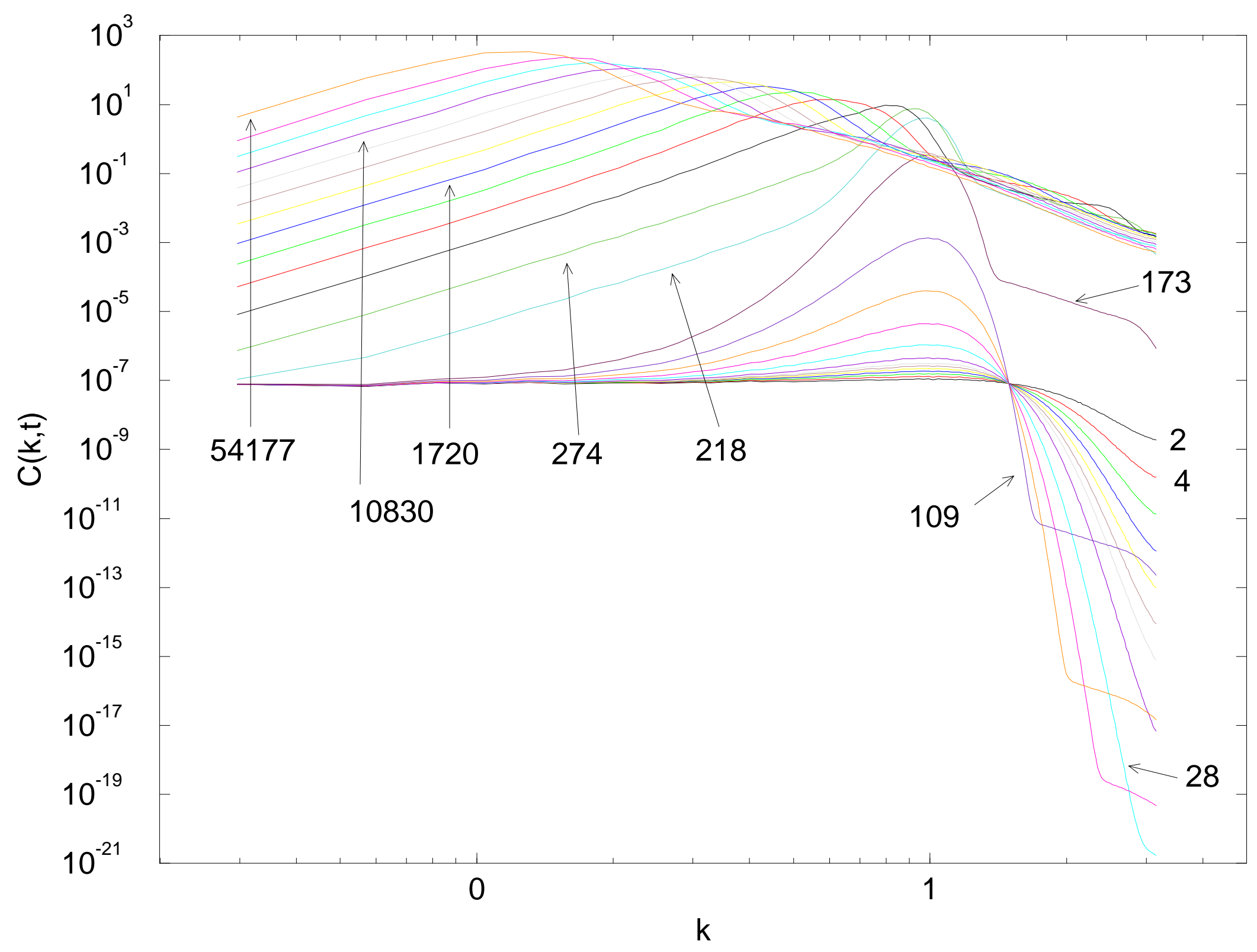




$$
N=1 \quad \Delta=8.3 \times 10^{-8}
$$

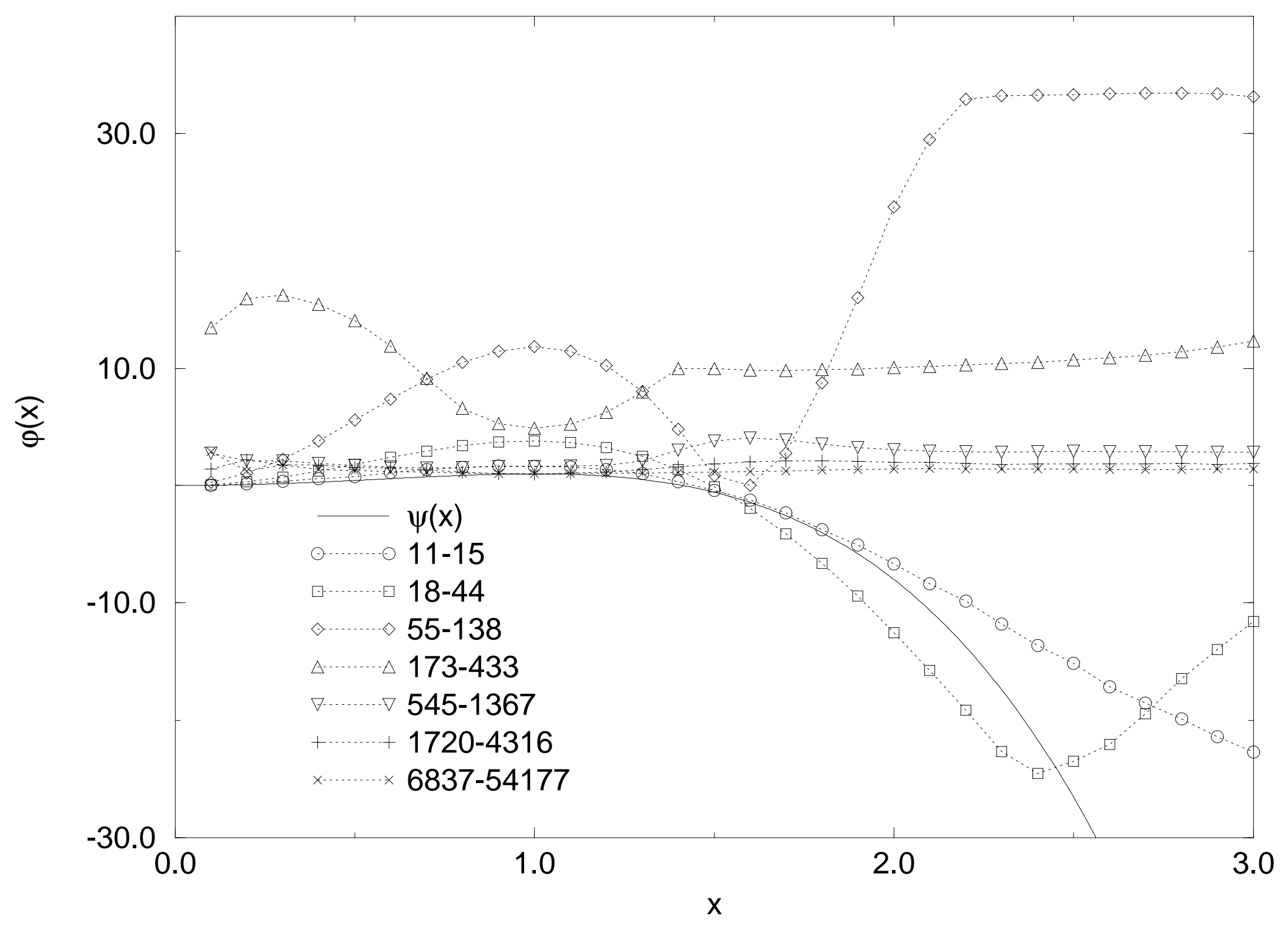




$$
N=1 \quad \Delta=2.083
$$

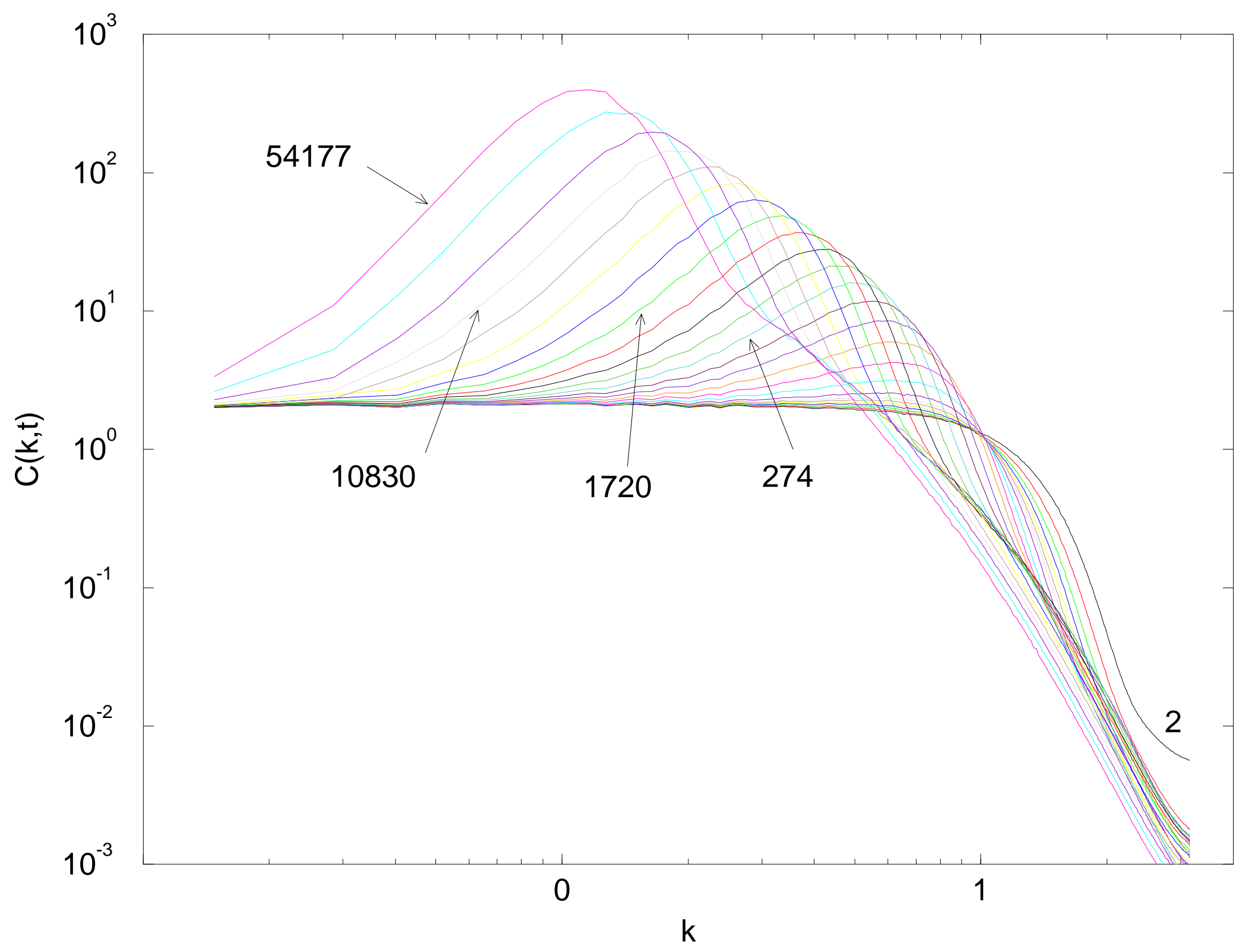


$N=1 \quad \Delta=2.083$

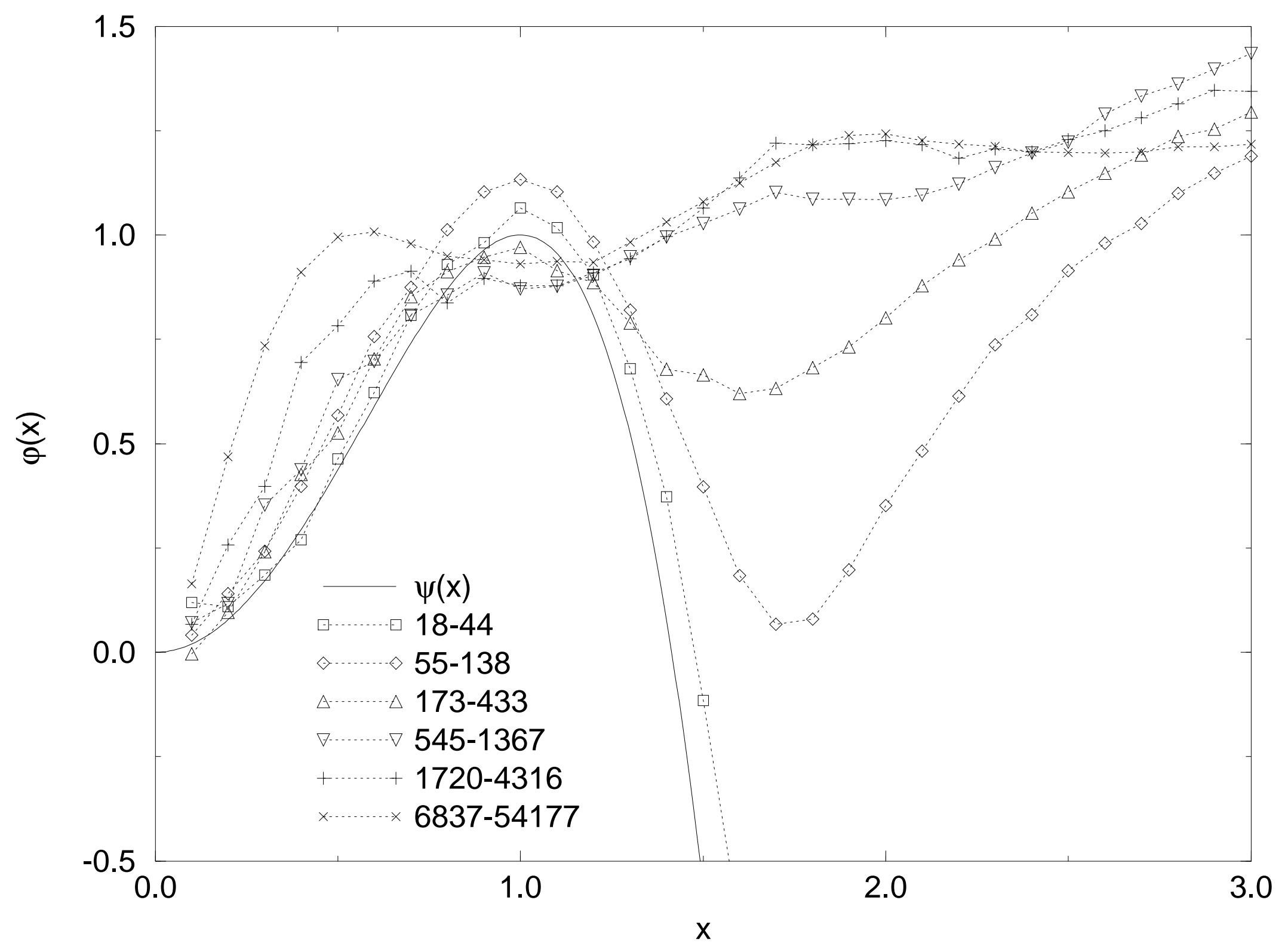




$$
N=4 \quad \Delta=2.083
$$

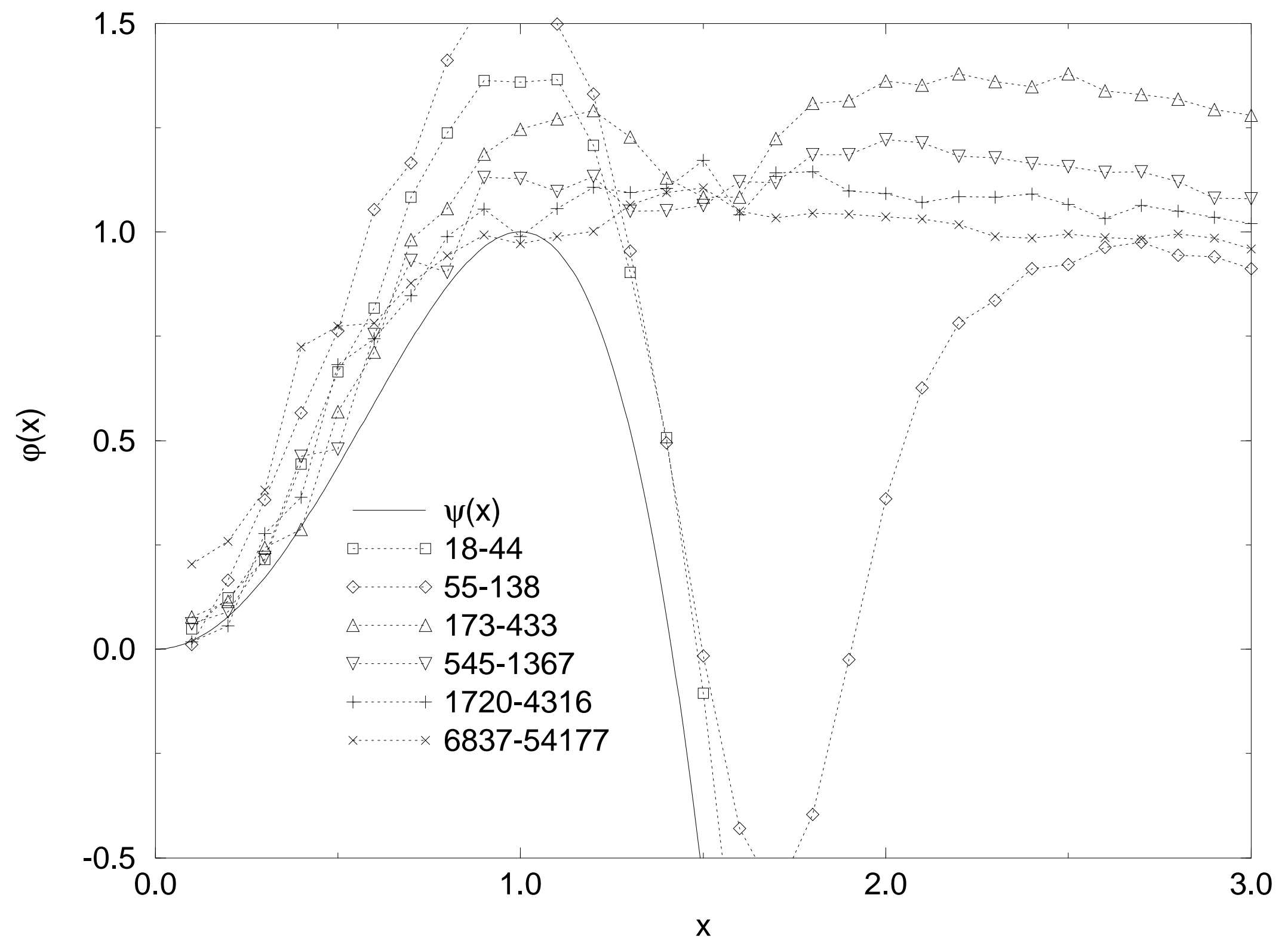




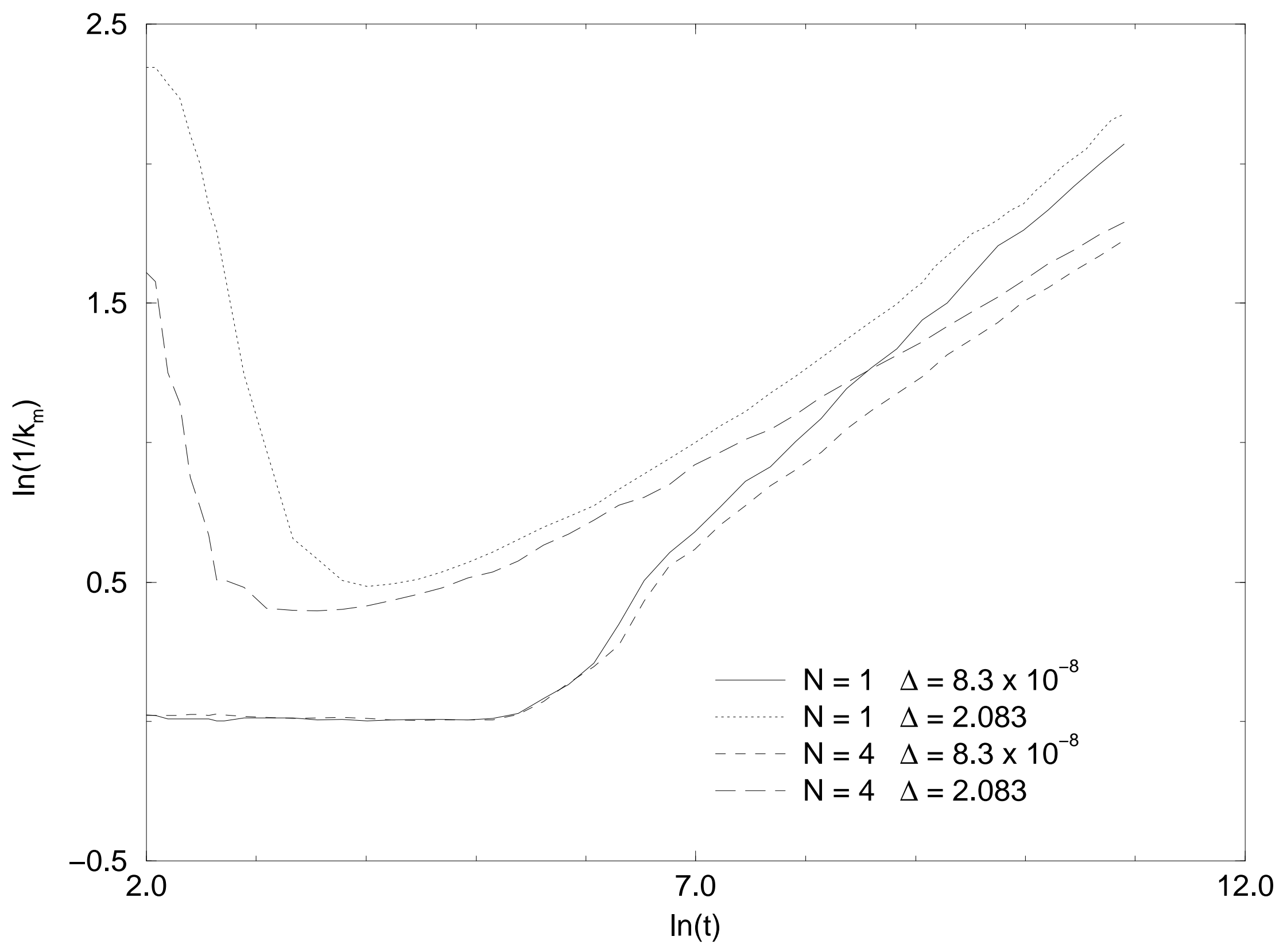

